\title{
Pattern of cortical activation during processing of aversive stimuli in traumatized survivors of war and torture
}

\section{Claudia Catani, Hannah Adenauer, Julian Keil, Hannah Aichinger, Frank Neuner}

\begin{abstract}
Posttraumatic stress disorder (PTSD) has been associated with an altered processing of threat-related stimuli. In particular, an attentional bias towards threat cues has been consistently found in behavioral studies. However, it is unclear whether increased attention towards threat cues translates into preferential processing as neurophysiological studies have yielded inconsistent findings. The aim of the present study was to investigate the neocortical activity related to the processing of aversive stimuli in patients with PTSD. 36 survivors of war and torture with PTSD, 21 Trauma Controls and 20 Unexposed Subjects participated in a visual evoked magnetic field study using flickering pictures of varying affective valence as stimulus material. Minimum norm source localization was carried out to estimate the distribution of sources of the evoked neuromagnetic activity in the brain. Statistical permutation analyses revealed reduced steady-state visual evoked field amplitudes over occipital areas in response to aversive pictures for PTSD patients and for Trauma Controls in comparison to unexposed subjects. Furthermore, PTSD patients showed a hyperactivation of the superior parietal cortex selectively in response to aversive stimuli, which was related to dissociative symptoms as well as to torture severity. The results indicate a different pattern of cortical activation driven by aversive stimuli depending on the experience of multiple traumatic events and PTSD.
\end{abstract}

\section{Catani $(\square) \cdot$ F. Neuner}

Clinical Psychology and Psychotherapy Unit,

Department of Psychology, University of Bielefeld,

33501 Bielefeld, Germany

e-mail: claudia.catani@uni-bielefeld.de

H. Adenauer - J. Keil $\cdot$ H. Aichinger

Department of Psychology, Center for Psychiatry Reichenau,

University of Konstanz, 78457 Konstanz, Germany
Whereas, a decreased visual processing of aversive stimuli seems to be associated with trauma exposure in general, the superior parietal activity might represent a specific process linked to the diagnosis of PTSD.

Keywords Posttraumatic stress disorder (PTSD) . Emotional processing . Steady-state visual evoked fields (SSVEF) · Magnetoencephalography (MEG)

\section{Introduction}

Current etiological models of posttraumatic stress disorder (PTSD) have identified the altered processing of threatening stimuli, in particular, stimuli that are reminiscent of the traumatic experiences, as a core feature of PTSD [14]. Consistent with this assumption, several studies on information processing in PTSD have consistently found an attentional bias to threat-related or trauma-relevant stimuli [8, 40, 41] in this population. In particular, it has been shown that PTSD patients are more easily distracted by task-irrelevant aversive stimuli, regardless of the relevance of these stimuli to the trauma. This finding seems to indicate a preferential detection and encoding of aversive stimuli in PTSD patients. However, it is unclear whether the increased task interference caused by distracting aversive stimuli is brought about by enhanced stimulus processing or by other cognitive processes that are triggered by the threatening stimuli.

The use of neurophysiological methods, in particular, electroencephalographic procedures with a high temporal resolution could help to understand alterations in the early processing of threatening cues in PTSD. Karl et al. [28] have recently reviewed studies examining event-related potentials in PTSD and concluded that this disorder is 
typically associated with alterations in the amplitude and latency of ERPs indicating that changes in information processing can accompany PTSD. More specifically, Attias and co-workers used an EEG oddball paradigm with Israeli combat veterans with and without PTSD $[1,6]$. They found indicators for a preferential processing of trauma-related stimuli within the first $500 \mathrm{~ms}$ after stimulus onset, as only PTSD patients showed enhanced P3 and N1 amplitudes to non-target combat-related pictures. A similar effect could be demonstrated for non-trauma related aversive stimuli [15]. However, research in this field is limited and inconsistent, as some studies failed to demonstrate enhanced event-related components towards traumatic targets in PTSD patients [42] or even found hyporesponsivity to threat cues in PTSD patients [16]. Findings from studies with high- and low-anxiety individuals might help to resolve these conflicting findings as these studies indicate that the relationship between anxiety and the attentional processing of aversive stimuli changes within the early processing stages $[22,48]$. High anxiety, and possibly also PTSD, seems to be related to a potentiation of the initial threat evaluation but an attenuation of the later cognitive processing aversive stimuli [22].

In the present study, we wanted to examine neural correlates of sustained cortical processing of aversive stimuli within the first second after stimulus presentation. In particular, we wanted to find out whether PTSD is related to alterations in the processing of threat related pictures in cortical areas that are related to visual attention and processing. We applied magnetoencephalography (MEG) to measure steady-state visual evoked fields (ssVEFs) during the presentation of standardized affective pictures varying with respect to arousal (high and low) and valence (pleasant and unpleasant). SsVEFs are the neuromagnetic counterparts of steady-state visual evoked potentials (ssVEPs) and represent a continuous brain response elicited by a repetitive visual stimulus presented at a predefined frequency (e.g. $10 \mathrm{~Hz}$ ). The ongoing cortical oscillatory neuromagnetic responses have the same fundamental frequency as the driving stimulus [46]. Past studies with healthy subjects have shown that highly arousing pictures generate greater ssVEFs or ssVEPs than neutral, low-arousing pictures mostly in occipital and parietal cortical networks $[30,32,43]$ indicating the allocation of attentional resources to stimuli according to their respective affective significance. Recently, a study by Moratti and co-workers has demonstrated that the pattern of sSVEFs elicited by emotional pictures can discriminate between patients with major depressive disorder and healthy control subjects [44].

In the present study, we were able to examine survivors of war and torture with a DSM-IV diagnosis of PTSD as well as a matched sample of refugees with similar ethnicities who had experienced traumatic experiences but did not fulfill criteria of PTSD. By comparing these two groups with healthy control subjects, we aimed at distinguishing between effects related to trauma severity and those related to the presence of the diagnosis of PTSD. Given the inconsistent findings of previous research on the processing of threat-related stimuli in PTSD, the main aim of the present study was to find out whether PTSD patients in comparison to trauma-affected subjects without PTSD and healthy individuals would show an increase or decrease in occipital-parietal neocortical activity related to the processing of aversive pictures, and whether the spatial pattern of activation would differ between groups. In agreement with existing research on neural correlates of affective visual processing [30,32,43], activation of visual cortical areas is seen as reflecting attention allocation towards emotionally and motivationally salient stimuli [36]. Finally, we wanted to explore whether trauma-related variables (such as the amount of previously experienced war and torture experiences) and clinical variables (such as PTSD severity, co-morbid depressive symptoms, and dissociative features) are related to source activity elicited by aversive stimuli.

\section{Materials and methods}

Subjects

Fifty war and torture-exposed participants and 20 comparison individuals with comparable ethnicities participated in the study. Trauma-exposed participants were asylum seekers with a history of persecution, war and torture who came for treatment or expert opinion to the Psychotrauma Research and Outpatient Clinic for Refugees, located at the Centre for Psychiatry, Reichenau, Germany. The trauma-exposed group was further subdivided according to PTSD diagnosis. The PTSD Group was composed of 36 subjects who fulfilled DSM-IV criteria for current PTSD, whereas 21 survivors of war and torture experiences did not meet PTSD criteria (Trauma Controls). Comparison participants were recruited by searching for migrants born in conflict regions with hardly any past traumatic experiences by announcements on campus bulletin boards (Unexposed Controls).

All subjects underwent an extensive clinical examination which included a thorough assessment of traumatic experiences by means of the vivo checklist of war, detention, and torture events [52]. Common traumatic experiences reported by refugees included bombings, seeing dead bodies in the street, being tortured in prison, or being beaten up by the police. A broader range of traumatic event types, including car accidents and criminal attacks, 
were assessed using the event checklist of the Clinician Administered PTSD Scale, CAPS [5]. Standardized clinical instruments were used for the assessment of DSM-IV diagnoses: the CAPS for the diagnosis and quantification of PTSD and related dissociative features, the Mini International Neuropsychiatric Interviews, MINI [49] for diagnoses of DSM-IV axis one disorders, the Hamilton Depression Rating Scale, HDRS [53] for determining the severity of depressive symptoms, and the Screening for Somatoform Symptoms-7, SOMS-7, [47] for the assessment of somatic problems.

The resulting demographic and clinical characteristics of the three groups and significant group differences are listed in Table 1. All participants provided written informed consent and the procedures were approved by the ethics committee of the University of Konstanz.

\section{Stimuli}

Seventy-five colored pictures were chosen on the basis of their normative ratings from the International Affective Pictures System [35]. Of these, 25 pictures presented unpleasant, events (e.g. mutilations, assaults, dead bodies etc.), 25 pictures presented pleasant events (e.g. sports, erotic couples, children, etc.) and 25 presented neutral events (e.g. neutral faces, household objects, etc.). ${ }^{1}$ Amongst the unpleasant pictures, the majority of stimuli was threat-related with about one-third depicting human assaults or aimed guns, another third showing mutilations or dead bodies. Scenes with soldiers or police officers were presented on three slides, and on another three, angry and sad faces were shown. The three categories differed significantly from each other in IAPS normative valence ratings (pleasant: 7.4, neutral: 4.9, unpleasant: 2.4). Normative arousal ratings did not differ for pleasant and unpleasant contents, but mean arousal levels for both emotional categories were significantly higher than for neutral contents (pleasant: 5.6, neutral: 2.9, unpleasant: 5.8). Brightness, contrast and color spectra of the stimuli were matched across picture categories. Pictures were presented using a video projector (JVC ${ }^{\mathrm{TM}}$, DLA-G11E) with a refresh rate of $100 \mathrm{~Hz}$ on a white plastic screen attached to the ceiling of the room. Pictures subtended a visual angle of $10^{\circ}$ horizontally and $8^{\circ}$ vertically to either side from the centre of the screen. In each trial, one picture

\footnotetext{
1 The numbers of IAPS pictures were as follows. Pleasant: 2190 , $2214,2215,2383,2440,2480,2516,2840,2850,5130,5510,5740$, $7035,7175,7217,7491,7500,7590,7595,7700,8190,5830,5660$, 4607,2209 . Neutral: 1722, 2030, 2058, 2165, 2216, 2311, 2340, 2345, $2352,4599,4608,4641,4653,4660,5260,5700,8185,8200,8380$, $8496,7490,7130,5390,2570,2410$. Unpleasant: $2120,2900,3181$, $3301,6190,6212,6250,6312,6540,6560,6831,6838,9040,9181$, $9400,9405,9415,9421,9433,9911,6821,3550,3530,2800,2053$.
}

was presented in a flickering mode of $10 \mathrm{~Hz}$ for $4 \mathrm{~s}$, resulting in 40 on/off cycles (same picture shown and not shown) of $100 \mathrm{~ms}$ each. The inter-trial interval varied randomly between 6 and $8 \mathrm{~s}$ and consisted of the presentation of a grey screen with a fixation cross to aid participants in maintaining gaze on the center of the screen.

\section{Procedure}

MEG recording was carried out within 1 week after clinical examination. Upon arriving at the laboratory, participants were familiarized with the MEG chamber and an informed consent form was signed. Handedness was determined using the Edinburgh Inventory (Oldfield 1971). For artifact control, the electro-oculogram and electrocardiogram were recorded. Subjects were seated in a magnetically shielded chamber and their head shapes were digitized with a Polhemus 3Space Fasttrack (Polhemus, Colchester, VT, USA). Five index points (left and right periauricular points, nasion, a pseudo- $\mathrm{Cz}$ and pseudo-inion point at the forehead) were determined to calculate the relative head position within the MEG helmet for source analysis. During MEG recording, a video camera monitored subjects' behavior and assured compliance throughout the experiment.

Participants were instructed to avoid eye movements and eye blinks during picture presentation. After MEG recordings, subjects rated each of the 75 affective pictures regarding emotional valence and arousal using the SelfAssessment Manikin self-report scale [7].

\section{MEG recording and data preprocessing}

MEG was recorded continuously and digitized at a rate of 678.17 Hz using a 148-channel whole head magnetometer (MAGNES $^{\text {TM }} 2500$ WH, 4D Neuroimage, San Diego, USA). A band-pass filter of $0.1-200 \mathrm{~Hz}$ was applied online. EOG and ECG were recorded with a SynAmps amplifier (Neuroscan ${ }^{\mathrm{TM}}$ ) using $\mathrm{Ag} / \mathrm{Cl}$ electrodes.

Offline, MEG data were visually inspected for movement artifacts. Global external noise and cardiac artifacts were corrected by means of procedures included in the MEG acquisition software package (Whole Head system software, version 1.2.5; 4D Neuroimaging). Eye artifacts were corrected using the algorithm implemented in BESA $^{\mathrm{TM}}$ software [3]. This method uses the vertical EOG to determine the topography of blinks based on the first component of a principle component analysis (PCA) of the average blink activity. The correction of blinks is then carried out by applying the surrogate MSEC methods as described by Berg and Scherg [3]. The MEG data were digitally band-pass filtered between 1 and $25 \mathrm{~Hz}$ (slopes: 6 and $24 \mathrm{~dB} /$ octave, respectively). Trials containing 
Table 1 Demographic and clinical characteristics of the three subject groups

\begin{tabular}{|c|c|c|c|}
\hline Variable & PTSD Group $(n=36)$ & Trauma Controls $(n=21)$ & Unexposed Group $(n=20)$ \\
\hline Mean age in years $(\mathrm{SD})$ & $33.6(10.0)$ & $29.9(9.6)$ & $27.8(9.4)$ \\
\hline Mean school educ. in years (SD) & $8.2(3.3)$ & $11.8(1.8)$ & $12(2.0)$ \\
\hline Females, $N(\%)$ & $15(38.5)$ & $13(61.9)$ & $11(55.0)$ \\
\hline \multicolumn{4}{|l|}{ Region of origin, $N(\%)$} \\
\hline Africa & $9(25.0)$ & $4(19.1)$ & $3(15.0)$ \\
\hline South Asia & 0 & $1(4.8)$ & 0 \\
\hline Caucasus & $2(5.6)$ & 0 & 0 \\
\hline Middle East & $22(61.1)$ & $11(52.4)$ & $5(25.0)$ \\
\hline Balcans & $3(8.3)$ & $5(23.8)$ & $12(60.0)$ \\
\hline Europe & 0 & 0 & 0 \\
\hline Safe asylum status, $N(\%)$ & $4(11.1)^{\mathrm{a}}$ & $16(76.2)^{\mathrm{b}}$ & $18(90.0)^{\mathrm{b}}$ \\
\hline \multicolumn{4}{|l|}{ Trauma load [mean number (SD)] } \\
\hline CAPS event types & $7.0(2.1)^{\mathrm{a}}$ & $5.9(1.4)^{\mathrm{a}}$ & $1.8(0.9)^{\mathrm{b}}$ \\
\hline War and torture event types & $10.9(5.7)^{\mathrm{a}}$ & $4.1(5.4)^{\mathrm{b}}$ & $0.6(1.3)^{\mathrm{b}}$ \\
\hline CAPS PTSD symptom score, mean (SD) & $80.5(16.6)^{\mathrm{a}}$ & $15.7(21.3)^{\mathrm{b}}$ & $3.5(8.8)^{\mathrm{b}}$ \\
\hline$N$ with dissociative symptoms (\%) & $12(35.3)^{\mathrm{a}}$ & $2(9.5)^{b}$ & $0^{\mathrm{b}}$ \\
\hline \multicolumn{4}{|l|}{ MINI diagnoses, $N(\%)$} \\
\hline MD current & $27(75)^{\mathrm{a}}$ & $2(9.5)^{\mathrm{b}}$ & $2(10.0)^{\mathrm{b}}$ \\
\hline Dysthymia & $3(8.3)$ & $2(9.5)$ & $2(10.0)$ \\
\hline Mean HDRS score (SD) & $25.6(7.7)^{\mathrm{a}}$ & $7.3(7.5)^{\mathrm{b}}$ & $3.9(6.7)^{\mathrm{b}}$ \\
\hline Mean SOMS score (SD) & $27.5(11.9)^{\mathrm{a}}$ & $8.7(10.4)^{b}$ & $4.9(7.2)^{\mathrm{b}}$ \\
\hline \multicolumn{4}{|l|}{ Medication, $N(\%)$} \\
\hline Pain killers & $20(55.6)^{\mathrm{a}}$ & $7(33.3)^{\mathrm{b}}$ & $2(10.0)^{\mathrm{b}}$ \\
\hline Anxiolytics & $2(5.6)$ & 0 & 0 \\
\hline Hypnotics & $3(8.3)$ & $1(4.8)$ & 0 \\
\hline Antidepressants & $8(22.2)$ & $2(9.5)$ & 0 \\
\hline Neuroleptics & $2(5.6)$ & $1(4.8)$ & 0 \\
\hline
\end{tabular}

Indices represent the results of pair-wise group comparisons using the Mann-Whitney $U$ tests for continuous variables and the $\chi^{2}$ test for dichotomous variables; different indices indicate significant differences on $P<0.05$

maximum amplitudes above $3.5 \mathrm{pT}$ were discarded from further analysis. Finally, MEG data were averaged for picture category (pleasant, neutral and unpleasant) over $5,000 \mathrm{~ms}$ (500 ms baseline, 4,000 ms stimulus presentation, 500 ms post stimulus).

For each category average, the $10-\mathrm{Hz}$ Fourier component was derived using a moving window averaging procedure [31]. To avoid contamination of results with the early event-related activity, the initial $500 \mathrm{~ms}$ of the picture presentation interval were excluded. The resulting 500 4,000 ms post stimulus part of each epoch was baselinecorrected (500 ms pre-stimulus) and used for further analysis. A 400-ms window containing four cycles of the $10-\mathrm{Hz}$ flickering stimuli was shifted in steps of $100 \mathrm{~ms}$ (one cycle) across the epoch, and the magnetic field data within the shifting windows in the time domain were further averaged. The resulting four cycles per category, subject, and MEG channel were submitted to the fast Fourier transform (FFT) technique [4]. The real and the imaginary parts of the $10-\mathrm{Hz}$ Fourier component were extracted for further analysis.

Source analysis

Using the Matlab-based software EMEGS $^{\oplus}$ [26], the distribution of likely generators of the neuromagnetic activity was estimated by calculating L2-minimum-norm solutions [18]. Calculation of the L2-minimum-norm was based on a one-shell spherical head model with 2 (azimuth and polar direction) $\times 197$ evenly distributed dipolar sources. A shell radius of $6 \mathrm{~cm}$ was chosen as the most preferable trade-off between depth sensitivity and spatial resolution [20]. The regularization parameter $\lambda$ was 0.02 and thus identical across all subjects and conditions. For visualization purposes, the estimated neural activities were projected onto the surface of a smoothened standard brain. Minimum norm estimation was calculated in the frequency domain by submitting the real and the imaginary parts of 
the $10 \mathrm{~Hz}$ Fourier component to the minimum norm estimates (MNE) analysis [24] and by using the root sum square of the 2 Fourier parts as an estimate of absolute power. As an example, mean activation towards unpleasant slides separately for each group is shown in Fig. 1.

\section{Statistical analysis}

The main aim of the statistical analysis of MNE data was to find differences between the three subject groups with respect to the processing of aversive stimuli. To determine the valence specificity of this effect, we repeated the same analysis for the pleasant stimulation. To control for different levels of overall stimulus-driven activity, source activity related to neutral pictures was subtracted from activity in response to aversive pictures ('unpleasant minus neutral' difference) and to pleasant pictures ("pleasant minus neutral' difference) for each subject. The resulting condition contrast maps were averaged across groups, resulting in two condition contrast maps per group. For both types of contrast map, three pair-wise statistical group comparisons (PTSD vs. Trauma Controls, PTSD vs. Unexposed and Trauma Controls vs. Unexposed) were carried out.

To cope with the high number of comparisons on dipole level without predetermining regions of interest, we applied permutation tests for the comparison of brain maps [29]. The advantage of the permutation test is that it does not require any a priori assumption about the distribution of the data, as it generates all possible permutations of the data to represent the data distribution. Given that permutation or randomization tests are able to cope with small and unequal samples in biomedical research, permutation tests are to be preferred to $t$ or $F$ tests when analyzing location differences [39]. A previous MEG study investigating the processing of high-arousing pictures in depressive patients has also used permutation analyses to show differences in arousal dependent modulation of source strengths between the depressive group and healthy controls [44].

For each comparison, we determined cut-off values for significant group differences of the maps at single dipole locations based on 1,000 draws. For each draw, the individual condition contrast maps were randomly exchanged between groups to generate data for a random group composition. The maximum as well as the minimum differences at all dipole locations obtained from each draw entered the distributions of 1,000 maximum and minimum difference values. The upper and the lower critical values were determined as the 25th $(P<0.025)$ lowest and highest values in this distribution.

In Table 2, upper and lower critical difference values for each pair-wise group comparison are listed. Values indicating a significant difference (smaller than the lower and higher than the upper critical values) were plotted onto the MNI brain to identify regions of interest (ROIs) associated with group differences in processing unpleasant as well as pleasant pictures. To allow a better understanding of group differences, $t$ tests for independent samples were conducted for each pair-wise comparison with the difference values at ROIs for "unpleasant minus neutral" and "pleasant minus neutral" as independent variables.

Valence and arousal ratings were evaluated using repeated-measures ANOVA with group as the between factor and with picture category as the within factor. Greenhouse-Geisser corrections of the degrees of freedom were applied where appropriate. Statistically significant interactions were investigated by using Tukey HSD test for post hoc evaluation of unequal sample sizes.
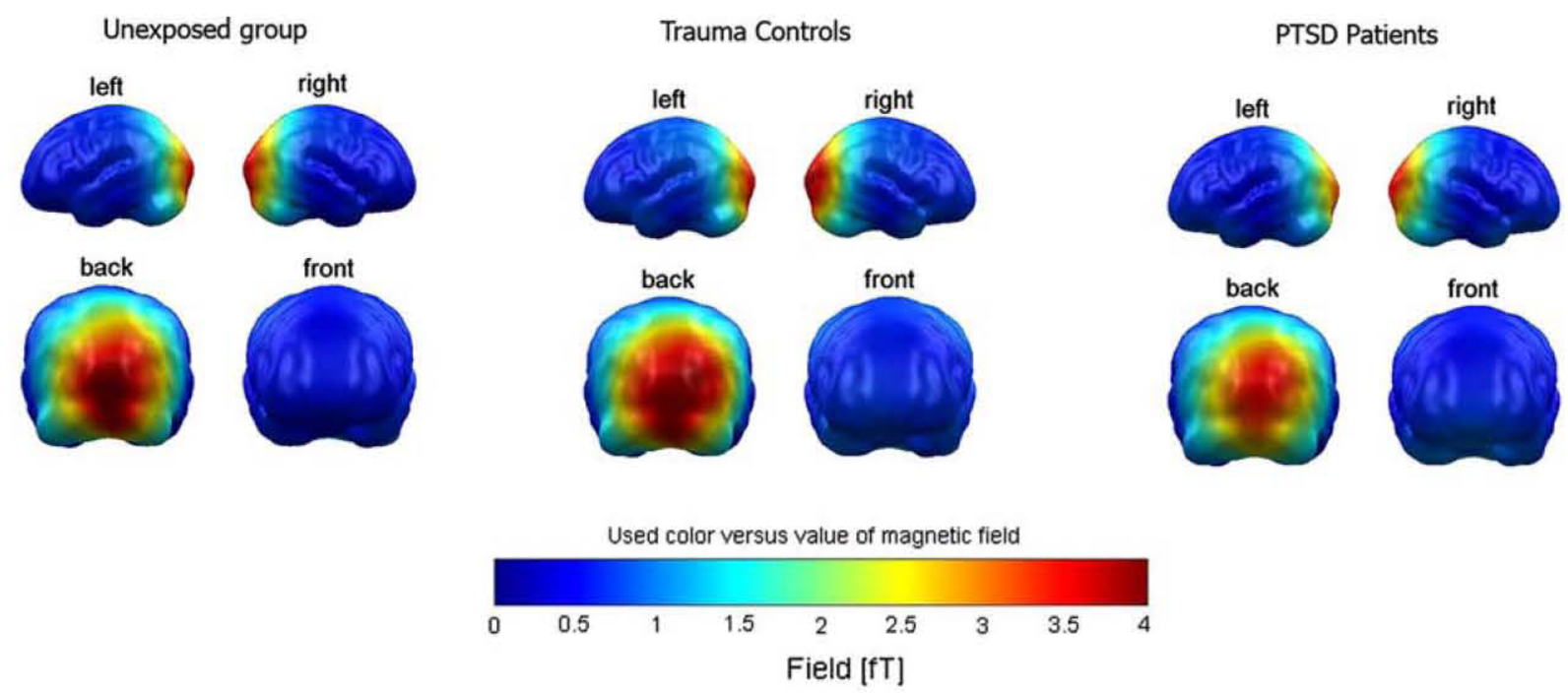

Fig. 1 Topographical brain maps showing cortical source activity following the presentation of unpleasant slides, separately for each group 
Table 2 Critical difference values with permutation $P<0.025$ for paired group comparisons

\begin{tabular}{lcl}
\hline & Lower critical value & Upper critical value \\
\hline Activation for pleasant pictures (pleasant minus neutral) & \\
Patients minus Unexposed Group & -0.24 & 0.23 \\
Patients minus Trauma Controls & -0.24 & 0.23 \\
Trauma Controls minus Unexposed Group & -0.27 & 0.26 \\
Activation for unpleasant pictures (unpleasant minus neutral) & \\
Patients minus Unexposed Group & -0.32 & 0.32 \\
Patients minus Trauma Controls & -0.32 & 0.31 \\
Trauma Controls minus Unexposed Group & -0.36 & 0.35 \\
\hline
\end{tabular}

In addition, exploratory analyses were carried out to investigate the relationship between subjective picture ratings, clinical variables, and cortical activation patterns separately for each picture category. Spearman rank correlation coefficients were used for these analyses. Correlations between clinical scores (CAPS, HDRS, and SOMS7) and activation towards unpleasant, neutral, and pleasant pictures at ROIs were carried out with a combined group of PTSD patients and Trauma Controls since the Unexposed participants scored consistently low on all clinical instruments. The analysis of correlations between trauma load (number of torture events, number of war events, number of events listed in the CAPS) and cortical activation was accomplished with trauma-exposed subjects who reported at least one experience in the respective category.

To test whether the presence of dissociative symptoms in PTSD patients might be related to activation patterns towards unpleasant or pleasant visual stimuli, we subdivided the PTSD sample into a subgroup who did not report any dissociative symptom on the CAPS $(n=22)$ and another subgroup with patients who reported at least one dissociative symptom (reduction in awareness, derealization or depersonalization) in the last 4 weeks $(n=12)$. It is important to note that these subgroups did not differ with respect to gender, to the number of traumatic, war or torture events experienced, or on PTSD symptom severity.

\section{Results}

\section{Self-assessment Manikin ratings}

As expected, arousal ratings differed between picture categories $[F(2,100)=188.4, P<0.001, \varepsilon=0.88]$ with aversive pictures being rated as more arousing compared to neutral $(P<0.001)$ as well as pleasant $(P<0.001)$ pictures. Pleasant pictures were also rated as more arousing than neutral pictures $(P<0.001)$. There was a significant interaction for group $\times$ category $[F(4,100)=4.25$, $P<0.005, \varepsilon=0.88$ ]. Figure 2 shows the different patterns of arousal ratings for the distinct stimulus conditions for each participant group.

With respect to valence rating, the expected main effect for 'picture category' was found $[F(2,92.9)=1051$, $P<0.001, \varepsilon=0.81]$. Overall, pleasant pictures were rated as more pleasant compared to neutral $(P<0.001)$ and unpleasant $(P<0.001)$ pictures. Neutral pictures were rated as more pleasant compared to unpleasant pictures $(P<0.001)$. There was an additional main effect for group $[F(2,57)=8.4, P<0.001]$ which is illustrated in Fig. 2 .

Minimum norm estimates (MNE) data

Permutation analyses revealed regions of interest (ROIs) with significant differences in pair-wise group comparisons. Figure 3 illustrates these group differences for the activation related to pleasant as well as to aversive visual stimuli. Both PTSD patients and Trauma Controls showed a reduced activation towards unpleasant pictures in centralleft occipital brain regions (amplitude mean of dipole sites 93, 116, 117, 118) compared to the Unexposed Sample (patients vs. unexposed: $t=-2.91, P<0.005$; trauma controls vs. unexposed: $t=-2.19 ; P<0.05$ ). In the Trauma Control Group, a similar pattern was visible for the activation towards pleasant stimuli where, again, trauma controls showed a significant reduction in mean amplitude compared to the unexposed participants $(t=-2.19$; $P<0.05$ ). In contrast, the difference between patients and unexposed subjects with respect to activation towards pleasant pictures did not yield statistical significance (see statistical maps in Fig. 3). However, patients differed significantly from trauma controls ( $t=3.69 ; P<0.005)$, showing a more pronounced activation towards pleasant pictures at occipital dipole site 116 .

With respect to the difference in activation between unpleasant and neutral pictures, permutation tests revealed the superior parietal cortex (dipole 15) as a further ROI, where PTSD patients showed an increased activation towards high arousing aversive stimuli compared to Trauma Controls $(t=2.12 ; P<0.05$ ). At this site, the 


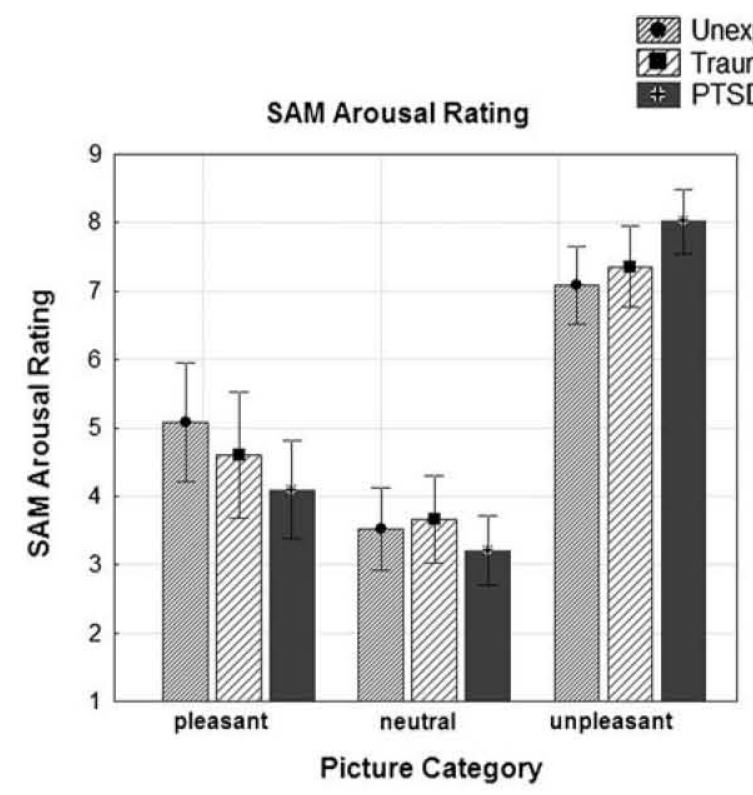

Fig. 2 SAM Arousal and Valence ratings of the three subject groups with error bars indicating 95\% confidence intervals. Post-hoc analyses showed that arousal ratings differed in relation to picture valence in the Unexposed Group. Here, unpleasant pictures were rated as more arousing compared to neutral $(P<0.001)$ as well as pleasant $(P<0.001)$ pictures. Pleasant pictures were rated as more arousing compared to neutral pictures $(P<0.005)$. Both, trauma controls and the patient group reported higher ratings for unpleasant pictures than

elevated response towards unpleasant pictures for PTSD patients when compared to the Unexposed Group approached significance $(t=-1.91 ; P=0.06)$. To provide a better overview, Fig. 4 depicts mean difference values for activation towards pleasant and unpleasant slides at ROIs identified by the permutation tests (occipital brain regions and superior parietal site).

Correlations between clinical variables, subjective affective ratings, and activation in occipital and superior-parietal brain regions

With respect to affective ratings, no group showed a significant relationship between SAM arousal or valence rating differences and corresponding activation patterns at occipital or superior parietal regions (ROIs). As can be seen in Table 3, activation towards unpleasant, neutral and pleasant pictures was also independent of clinical scores on the CAPS, the HDRS, and the SOMS-7 with respect to both regions of interests. A significant positive correlation was found between torture severity (the amount of torture events ever experienced) and SSVEF source amplitudes in response to unpleasant pictures over the superior parietal cortex (Rank correlation $r=0.37 ; P<0.05$ ). A graphical illustration of this relationship is presented in Fig. 5.

\section{SAM Valence Rating}

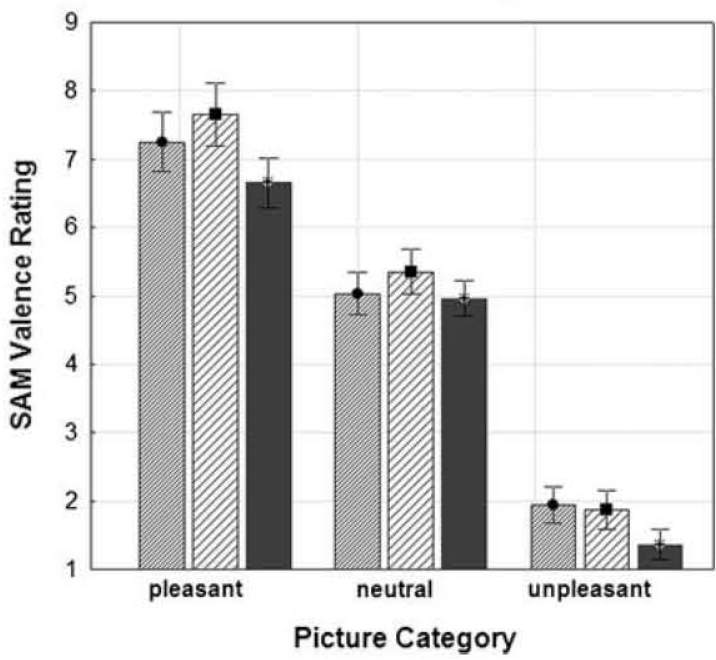

for neutral ( $P<0.001$ for both comparisons) as well as for pleasant ( $P<0.001$ for both comparisons) pictures. However, these two latter groups did not differ between neutral and pleasant picture with respect to their subjective arousal rating. With respect to stimulus valence, PTSD patients rated pictures in general as less pleasant as compared to Trauma Controls $(P<0.005)$ as well as to the Unexposed Group $(P<0.05)$. There was no difference between the non-PTSD samples with respect to their subjective valence ratings

The comparison between PTSD patients with dissociative symptoms and those without showed a significant difference $(t=-2.37 ; P<0.05$ ) with respect to SSVEF source amplitudes in response to unpleasant pictures over the superior parietal cortex (dipole 15). PTSD patients with current dissociative symptoms showed a higher activity towards unpleasant slides (mean activity $=0.014 \mathrm{nA} / \mathrm{mm}$ ) compared to PTSD patients without those specific features (mean activity $=0.48 \mathrm{nA} / \mathrm{mm}$.

\section{Discussion}

The present findings point towards a deviant processing of aversive pictures and, to a smaller extent, of high arousing pleasant pictures in individuals traumatized by war and torture. Consistent with previous research, we found higher activation over occipital areas towards high-arousing pictures in healthy subjects. Studies examining ssVEF as well ssVEP changes as a function of emotional arousal have shown greater amplitudes in regions involving the occipital cortices and temporo-parietal cortices [30, 32, 43]. These findings are typically discussed within the framework of selective attention, suggesting that more attentional resources are allocated to external stimuli according to 
Group comparison 'PTSD Patients minus Unexposed group'

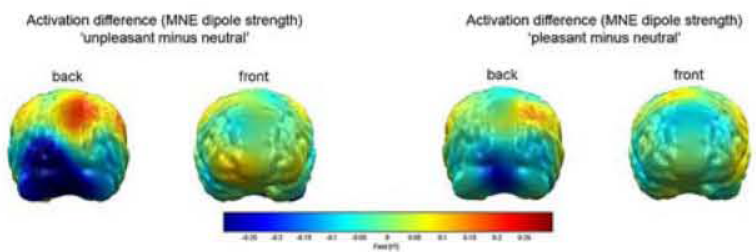

Statistically significant differences (exceeding critical values)

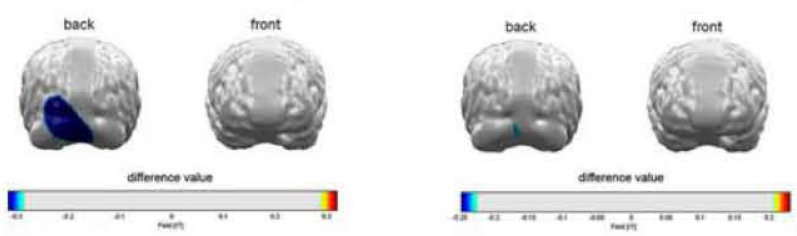

Group comparison 'Trauma Controls minus Unexposed group'

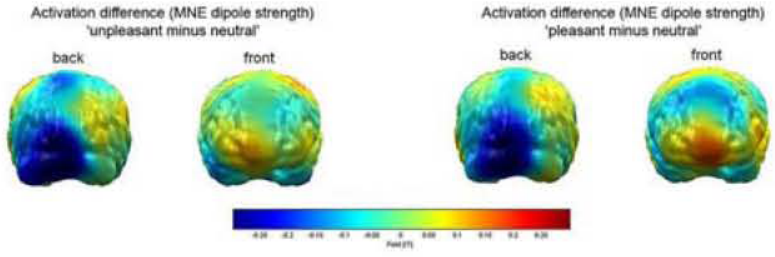

Statistically significant differences (exceeding critical values)
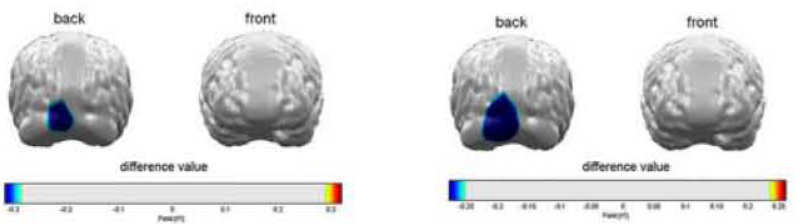

Group comparison 'PTSD Patients minus Trauma controls"

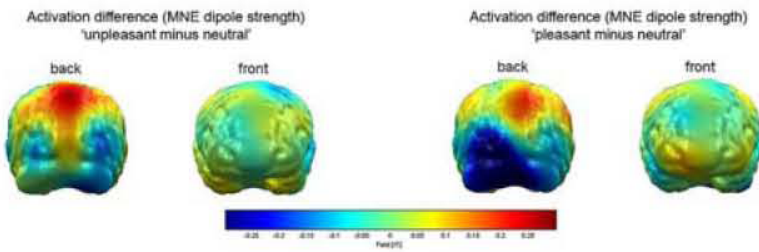

Statistically significant differences (exceeding critical values)
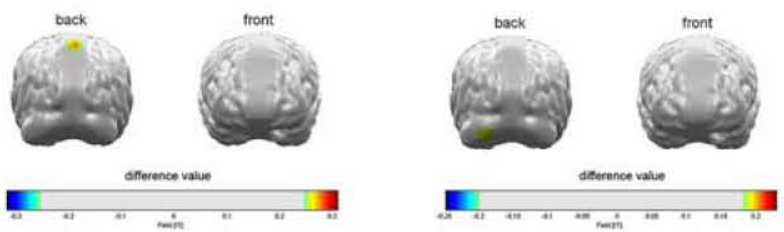

Fig. 3 Brain maps showing significant group differences with respect to cortical source activation towards unpleasant (left) and pleasant (right) picture content. For each of the three group comparisons, the upper series of brain plots show overall activation differences. The lower row of each comparison depicts those cortical sources which indicate significant differences between groups as calculated in the permutation analysis their affective significance to enhance sensory processing of relevant information $[21,36]$. In the present study, the preferential visual encoding of aversive stimuli was significantly reduced in the central-left occipital cortex in both PTSD patients and Trauma Controls. For PTSD patients, this hypoactivation in comparison to healthy controls was specific for aversive stimuli whereas the Trauma Control group showed similarly reduced steady-state responses in primary visual areas for pleasant pictures as well. The current finding of a decrease of the neurophysiological response towards aversive stimuli is in line with previous studies showing reduced ERP components to angry faces [16] and traumatic words [33]. In both of these studies, however, reduced event-related activity was found for aversive as well as for neutral stimuli indicating a generally reduced cortical activity in PTSD. Based on these findings, the authors suggested the presence of an adaptive sensorygating mechanism in PTSD [16]. In the present study, the assumption of a more general sensory-gating mechanism seems to be true for individuals who have experienced trauma without developing PTSD. The significant reduction of the activation towards both, pleasant and aversive high arousing pictures in the Trauma Control Group is in line with the assumption of a cortical inhibition mechanism in response to highly emotional cues that might represent a protective factor for the development of PTSD after trauma experiences.

In contrast to the Trauma Controls, PTSD patients showed a selective and more pronounced decrease of their ssVEFs in primary visual areas only in response to aversive pictures when compared to neutral ones. Applying Lang's theory of motivated attention [34, 36], one could assume that PTSD should be associated with an increase in sensory processing of aversive stimuli given their particular salience and motivational relevance. The cortical response pattern of PTSD patients in the present study, however, does not support this assumption but rather suggests that visual attention is lowered towards stimuli which are aversive and threat-related. A possible explanation could be that in PTSD patients, aversive pictures with such explicit contents as war and attack scenes are immediately categorized as a threat and do not require sustained visual processing to identify further emotionally relevant information. In this regard, studies using a rapid serial visual presentation method with healthy subjects have shown that affective processing of pictures occurs with great rapidity with high-arousing emotional slides activating occipitoparietal areas as early as $150 \mathrm{~ms}$ after stimulus onset [2527]. In the future, similar studies should be carried out with PTSD patients in order to distinguish between rapidly 
A Occipital dipole sites $(93,116,117,118)$

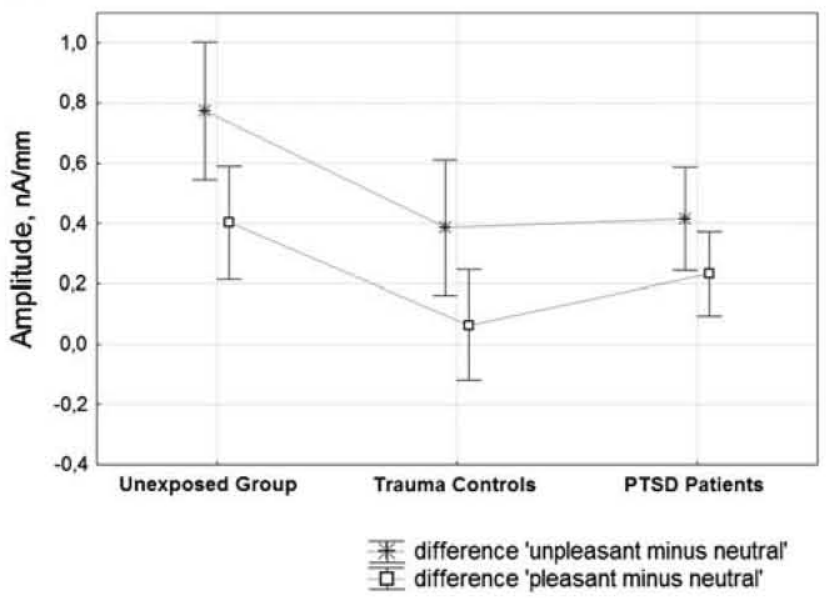

B

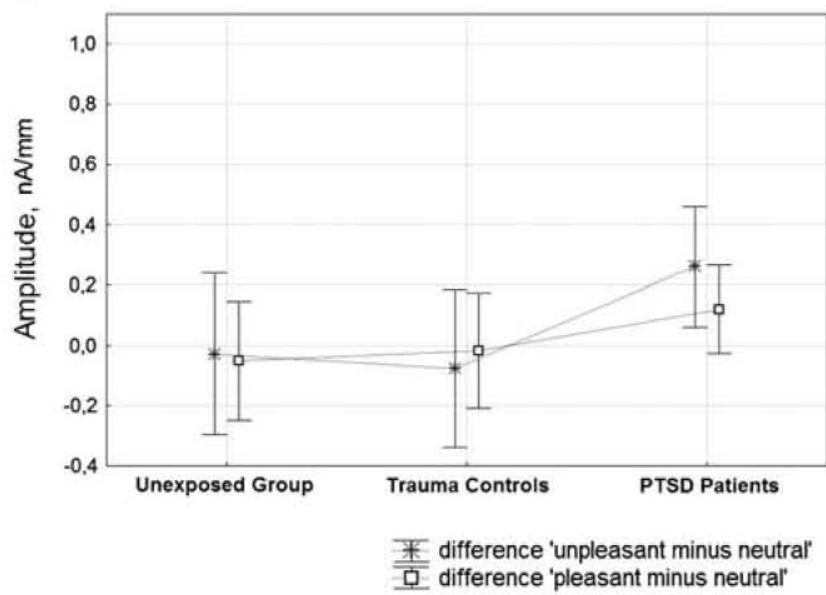

Fig. 4 Groups mean activation (in $\mathrm{nA} / \mathrm{mm}$ ) towards high arousing unpleasant and pleasant slides at ROIs (a Mean activation difference at four occipital dipole sites, b Activation differences at superior parietal site). Error bars indicate $95 \%$ confidence intervals

Table 3 Spearman rank correlations between trauma load and clinical variables and activation towards emotional pictures across all traumaexposed participants, separately for each affective category

\begin{tabular}{lcccccc}
\hline & $\begin{array}{l}\text { Occipital } \\
\text { activity } \\
\text { (pleasant) }\end{array}$ & $\begin{array}{l}\text { Occipital } \\
\text { activity } \\
\text { (neutral) }\end{array}$ & $\begin{array}{l}\text { Occipital } \\
\text { activity } \\
\text { (unpleasant) }\end{array}$ & $\begin{array}{l}\text { Superior } \\
\text { parietal } \\
\text { activity } \\
\text { (pleasant) }\end{array}$ & $\begin{array}{l}\text { Superior } \\
\text { parietal } \\
\text { activity } \\
\text { (neutral) }\end{array}$ & $\begin{array}{l}\text { Superior } \\
\text { parietal } \\
\text { activity } \\
\text { (unpleasant) }\end{array}$ \\
\hline Nr of traumatic events (CAPS) & -0.16 & -0.16 & -0.19 & -0.10 & -0.14 & 0.04 \\
Nr of war events & 0.06 & -0.05 & 0.06 & 0.10 & -0.02 & -0.06 \\
Nr of torture events & 0.12 & 0.12 & 0.15 & 0.15 & 0.29 & $0.36^{*}$ \\
PTSD severity score (CAPS) & -0.08 & -0.07 & -0.08 & -0.15 & -0.19 & -0.08 \\
Re-experiencing score & 0.04 & 0.04 & 0.02 & -0.19 & -0.24 & 0.02 \\
Avoidance score & -0.20 & -0.20 & -0.21 & -0.12 & -0.17 & -0.21 \\
Hyperarousal score & -0.07 & -0.07 & -0.07 & -0.23 & -0.16 & -0.07 \\
Depression Score (HDRS) & -0.01 & 0.01 & 0.03 & -0.12 & -0.19 & -0.08 \\
Nr of somatic complaints (SOMS-7) & -0.13 & -0.11 & -0.06 & -0.10 & -0.17 & -0.10 \\
\hline
\end{tabular}

* Correlations significant on $P<0.05$

occurring discriminative cortical mechanisms and sustained attentional processing of emotional pictures. In line with this thinking, a recent ERP study showed an enhanced initial threat evaluation but an attenuation of later cognitive processing of fearful faces in individuals with high anxiety compared to low-anxiety subjects [22].

In all subject groups, ssVEF amplitudes towards unpleasant or pleasant pictures at occipital sites were not significantly related to any clinical score (CAPS, HDRS or SOMS-7) or to trauma severity. Also, the subjective evaluation of pictures did not yield a similar pattern when compared with brain responses. To our knowledge, no analogous data with PTSD patients have been published so far, but studies with other clinical populations have shown that physiological reactivity during emotion processing and subjective evaluation of the stimuli can dissociate $[17,44]$.
The finding that both PTSD patients and Trauma Controls rated pleasant pictures as less arousing compared with the Unexposed Group could be related to the higher depression scores in the two trauma-exposed samples. In fact, previous studies on depressive patients have shown diminished emotional responses to pleasant stimuli $[50,51]$. The finding of the present study that PTSD patients rated all pictures as less pleasant, might therefore reflect the fact that the majority of the patients had co-morbid Major Depression.

In addition to the activation pattern in occipital brain areas, permutation analyses yielded a PTSD specific response towards aversive stimuli in the superior parietal cortex (SPC). At this site, we found a significantly augmented steady-state response towards unpleasant pictures in PTSD patients compared to Trauma Controls and, on a 
Fig. 5 Scatterplot illustrating the relationship between the number of torture events and superior parietal activity (dipole $15)$ towards aversive slides $(N=37$ participants with at least one torture experience are included)

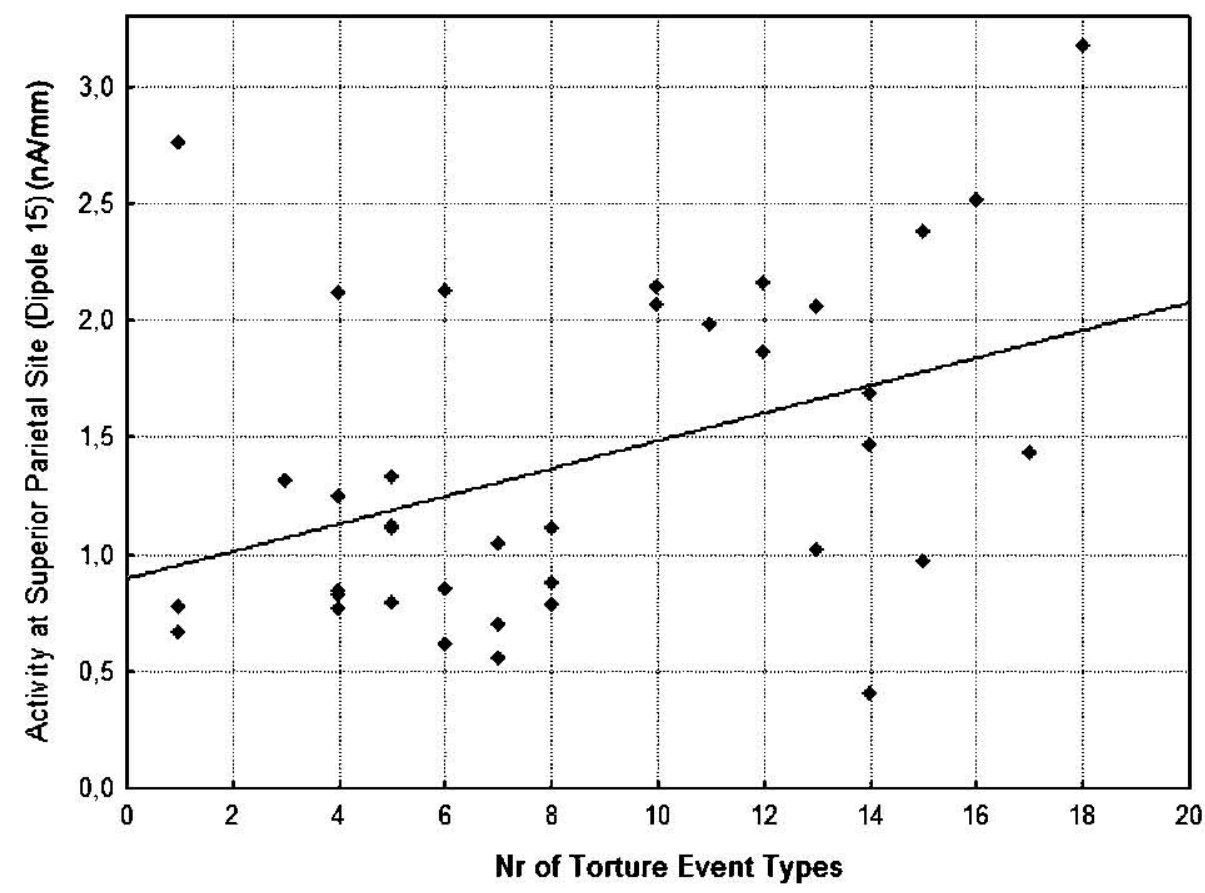

statistical trend level, to the Unexposed Group. In humans, the SPC is known to play a role in spatial information processing $[13,19]$ and contributes to top-down attentional processes on episodic memory [9]. Results from a study measuring regional cerebral blood flow in PTSD patients while performing a visuoverbal task show a higher activation of the superior parietal lobule in patients compared to healthy subjects [12]. The authors suggested an increased reliance of working memory function on the visuospatial coding of information in PTSD Patients.

Intriguing findings about a specific involvement of the SPC in dissociative symptoms in traumatized individuals emerged from neuroimaging studies with PTSD subjects [37, 38] and patients with Borderline-Personality Disorder (BPD) who had been exposed to childhood abuse [23]. Lanius and co-workers found increased activity of the SPC in PTSD patients with trauma-related dissociative states during script-driven imagery. The enhanced SPC activity did differentiate PTSD patients with a dissociative response to the traumatic script-driven imagery and those who had a flashback/reliving response to the script [37]. In line with these findings, in the present study, PTSD patients with current dissociative symptoms showed a higher superior parietal activation towards aversive stimuli compared to non-dissociative PTSD patients. In addition, we found a positive correlation between the amount of torture events ever experienced by trauma-exposed subjects and steady-state amplitudes in the SPC in response to aversive pictures. A possible explanation for this finding might be that torture experiences represent a massive and often chronic form of traumatization that typically does not only lead to PTSD but to a variety of other clinical disorders including dissociative symptoms $[2,10,11]$. Emphasizing the important role of dissociative features in survivors of torture, a previous study examining generators of cortical slow waves reported brain abnormalities in torture survivors with PTSD that were associated to the amount of dissociative experiences [45]. Given that, in the present study, no other clinical score was significantly related to SPC hyperactivation towards aversive stimuli, our data suggest a specific involvement of the superior parietal lobe in the processing of aversive cues which is mediated by the amount of torture experiences and the presence of dissociative symptoms in PTSD individuals. However, results of the correlational analyses should be interpreted with caution given that we had no a-priori hypotheses for the relationship between clinical data and cortical activation patterns and did not correct for multiple comparisons.

Another limitation of the present study is that group differences cannot be solely attributed to PTSD status because there were substantial differences between groups, in particular, with respect to comorbid depression as well as slight differences regarding the use of antidepressants. However, this problem is inherent in most PTSD research because PTSD is commonly associated with other disorders. Also, a recent MEG study with medicated depressive patients has reported a very different pattern of abnormalities during processing of high arousing stimuli [44] compared to the present outcome for PTSD patients, supporting the assumption that a possible confounding effect of depressive symptoms cannot explain group differences found in the present work. As a methodological constraint, 
the present study employed a homogenous sphere as a head model for the MNE. Thus, cortical areas involved in generation of the signal were inferred from localizations on a shell. Still, we consider this approach more accurate than simply inferring activation of cortical areas from sensor topographies. Ideally, future studies should utilize realistic head models derived from subjects' individual structural MRIs to obtain more exact identification of brain regions.

Based on the results of the present study, we cannot determine whether the occipital hypoactivation in PTSD patients reflects a specific response towards trauma-related stimuli or towards high arousing aversive pictures in general. As the majority of pictures showed human attacks, war scenes or injuries, most of them might have triggered memories related to the individual traumatic experiences. It remains a challenging task for future investigations to disentangle these responses. Ideally, studies should be carried out with a wide range of unpleasant pictures including trauma-related and non-trauma-related slides being shown to patients affected by a very specific trauma type (e.g. only car accident victims).

In conclusion, we found that the response pattern of the occipital cortex as well as the superior parietal cortex in response to aversive stimuli discriminates between nontraumatized subjects as well as victims of war and torture with and without PTSD. The occipital hypoactivation of the trauma survivors is independent of psychopathology and might represent an adaptive adjustment of the brain to live in a threatening environment. It can be speculated that this form of inner shut-down of the visual system is the cost for the rapid allocation of resources towards a defensive reaction immediately after the classification of a cue as threatening. However, it seems that the human cortical attention system can be shaped by life experiences in an adaptive or a pathological way, as we found an opposite response pattern of the superior parietal cortex for trauma survivors with and without PTSD. The pathological way of cortical attention allocation after aversive stimuli involves hyperactivation of the SPC and is related to more traumatic events, more PTSD symptoms and the presence of dissociative symptoms. Future studies could try to disentangle the predictors of the pathological attention pathway and try to change this mechanism through behavioral training.

\footnotetext{
Acknowledgments We are grateful to all subjects who took part in the study. Research was supported by the Deutsche Forschungsgemeinschaft (DFG) and the European Refugee Fund (EFF). The authors would like to thank Heike Riedke for logistical support, the outpatient clinic staff for clinical support, Ursula Lommen for helping with MEG data acquisition, Stephan Moratti and Christian Wienbruch for technical advice, Sonja Haas and Steivan Pinösch for helping with entry and administration of data, and Katy Robjant for editing.
}

Conflict of interest statement Authors declare they have no conflict of interest. The corresponding author had full access to all of the data in the study and takes responsibility for the integrity of the data and the accuracy of the data analysis.

\section{References}

1. Attias J, Bleich A, Furman V, Zinger Y (1996) Event-related potentials in post-traumatic stress disorder of combat origin. Biol Psychiatry 40:373-381

2. Basoglu M, Jaranson J, Mollica R, Kastrup M (2001) Torture and mental health: a research overview. In: Garrity T, Keane T, Tuma F (eds) The mental health consequences of torture. Plenum, New York, pp 35-62

3. Berg P, Scherg M (1994) A multiple source approach to the correction of eye artifacts. Electroencephalogr Clin Neurophysiol 90:229-241

4. Bickford RG, Fleming N, Billinger T (1971) Compression of EEG data. Trans Am Neurol Assoc 96:118-122

5. Blake DD, Weathers FW, Nagy LM, Kaloupek DG, Gusman FD, Charney DS, Keane TM (1995) The development of a ClinicianAdministered PTSD Scale. J Trauma Stress 8:75-90

6. Bleich AV, Attias J, Furman V (1996) Effect of repeated visual traumatic stimuli on the event related $\mathrm{P} 3$ brain potential in posttraumatic stress disorder. Int J Neurosci 85:45-55

7. Bradley MM, Lang PJ (1994) Measuring emotion: the SelfAssessment Manikin and the Semantic Differential. J Behav Ther Exp Psychiatry 25:49-59

8. Buckley TC, Blanchard EB, Neill WT (2000) Information processing and PTSD: a review of the empirical literature. Clin Psychol Rev 20:1041-1065

9. Cabeza R (2008) Role of parietal regions in episodic memory retrieval: the dual attentional processes hypothesis. Neuropsychologia 46:1813-1827

10. Campbell TA (2007) Psychological assessment, diagnosis, and treatment of torture survivors: a review. Clin Psychol Rev $27: 628-641$

11. Carlson EB, Rosser-Hogan R (1994) Cross-cultural response to trauma: a study of traumatic experiences and posttraumatic symptoms in Cambodian refugees. J Trauma Stress 7:43-58

12. Clark CR, McFarlane AC, Morris P, Weber DL, Sonkkilla C, Shaw M, Marcina J, Tochon-Danguy HJ, Egan GF (2003) Cerebral function in posttraumatic stress disorder during verbal working memory updating: a positron emission tomography study. Biol Psychiatry 53:474-481

13. Corbetta M, Shulman GL, Miezin FM, Petersen SE (1995) Superior parietal cortex activation during spatial attention shifts and visual feature conjunction. Science 270:802-805

14. Ehlers A, Clark DM (2000) A cognitive model of posttraumatic stress disorder. Behav Res Ther 38:319-345

15. Ehlers CL, Hurst S, Phillips E, Gilder DA, Dixon M, Gross A, Lau P, Yehuda R (2006) Electrophysiological responses to affective stimuli in American Indians experiencing trauma with and without PTSD. Ann NY Acad Sci 1071:125-136

16. Felmingham KL, Bryant RA, Gordon E (2003) Processing angry and neutral faces in post-traumatic stress disorder: an eventrelated potentials study. NeuroReport 14:777-780

17. Gehricke J, Shapiro D (2000) Reduced facial expression and social context in major depression: discrepancies between facial muscle activity and self-reported emotion. Psychiatry Res 95:157-167 
18. Hamalainen MS, Ilmoniemi RJ (1994) Interpreting magnetic fields of the brain: minimum norm estimates. Med Biol Eng Comput 32:35-42

19. Han S, Jiang Y (2004) The parietal cortex and attentional modulations of activities of the visual cortex. Neuroreport 15:22752280

20. Hauk O (2004) Keep it simple: a case for using classical minimum norm estimation in the analysis of EEG and MEG data. Neuroimage 21:1612-1621

21. Hillyard SA, Anllo-Vento L (1998) Event-related brain potentials in the study of visual selective attention. Proc Natl Acad Sci USA 95:781-787

22. Holmes A, Nielsen MK, Green S (2008) Effects of anxiety on the processing of fearful and happy faces: an event-related potential study. Biol Psychol 77:159-173

23. Irle E, Lange C, Weniger G, Sachsse U (2007) Size abnormalities of the superior parietal cortices are related to dissociation in borderline personality disorder. Psychiatry Res 156:139-149

24. Jensen O, Vanni S (2002) A new method to identify multiple sources of oscillatory activity from magnetoencephalographic data. Neuroimage 15:568-574

25. Junghöfer M, Bradley MM, Elbert TR, Lang PJ (2001) Fleeting images: a new look at early emotion discrimination. Psychophysiology 38:175-178

26. Junghöfer M, Peyk P (2004) Analyse und Visualisierung von Hirnstrom- und Hirnmagnetfeld-Messungen [analysis of electrical potentials and magnetic fields of the brain]. Matlab Select 2:24-28. EMEGS software is freely available at http://www. emegs.org/

27. Junghofer M, Sabatinelli D, Bradley MM, Schupp HT, Elbert TR, Lang PJ (2006) Fleeting images: rapid affect discrimination in the visual cortex. NeuroReport 17:225-229

28. Karl A, Malta LS, Maercker A (2006) Meta-analytic review of event-related potential studies in post-traumatic stress disorder. Biol Psychol 71:123-147

29. Karniski W, Blair RC, Snider AD (1994) An exact statistical method for comparing topographic maps, with any number of subjects and electrodes. Brain Topogr 6:203-210

30. Keil A, Gruber T, Muller MM, Moratti S, Stolarova M, Bradley MM, Lang PJ (2003) Early modulation of visual perception by emotional arousal: evidence from steady-state visual evoked brain potentials. Cogn Affect Behav Neurosci 3:195-206

31. Keil A, Stolarova M, Heim S, Gruber T, Müller MM (2003) Temporal stability of high-frequency brain oscillations in the human EEG. Brain Topogr 16:101-110

32. Kemp AH, Gray MA, Eide P, Silberstein RB, Nathan PJ (2002) Steady-state visually evoked potential topography during processing of emotional valence in healthy subjects. NeuroImage $17: 1684-1692$

33. Kounios J, Litz B, Kaloupek D, Riggs D, Knight J, Weathers F, Anderson JE, Keane T (1997) Electrophysiology of combatrelated PTSD. Ann NY Acad Sci 821:504-507

34. Lang PJ, Bradley MM, Cuthbert BN (1998) Emotion, motivation, and anxiety: brain mechanisms and psychophysiology. Biol Psychiatry 44:1248-1263

35. Lang PJ, Bradley MM, Cuthbert BN (2005) International Affective Picture System (IAPS): affective ratings of pictures and instruction manual. University of Florida, Gainesville

36. Lang PJ, Bradley MM, Cuthbert BN (1997) Motivated attention: affect, activation and action. In: Lang PJ, Simons RF, Balaban
MT (eds) Attention and orienting: sensory and motivational processes. Erlbaum, Mahwah

37. Lanius RA, Williamson PC, Bluhm RL, Densmore M, Boksman K, Neufeld RW, Gati JS, Menon RS (2005) Functional connectivity of dissociative responses in posttraumatic stress disorder: a functional magnetic resonance imaging investigation. Biol Psychiatry $57: 873-884$

38. Lanius RA, Williamson PC, Boksman K, Densmore M, Gupta M, Neufeld RW, Gati JS, Menon RS (2002) Brain activation during script-driven imagery induced dissociative responses in PTSD: a functional magnetic resonance imaging investigation. Biol Psychiatry $52: 305-311$

39. Ludbrook J, Dudley H (1998) Why permutation tests are superior to $t$ and $F$ tests in biomedical research. The American Statistician $52: 127-132$

40. McNally RJ, Clancy SA, Schacter DL, Pitman RK (2000) Cognitive processing of trauma cues in adults reporting repressed, recovered, or continuous memories of childhood sexual abuse. J Abnorm Psychol 109:355-359

41. McNally RJ, Kaspi SP, Riemann BC, Zeitlin SB (1990) Selective processing of threat cues in posttraumatic stress disorder. $\mathbf{J} \mathrm{Ab}-$ norm Psychol 99:398-402

42. Metzger LJ, Orr SP, Lasko NB, Pitman RK (1997) Auditory event-related potentials to tone stimuli in combat-related posttraumatic stress disorder. Biol Psychiatry 42:1006-1015

43. Moratti S, Keil A, Stolarova M (2004) Motivated attention in emotional picture processing is reflected by activity modulation in cortical attention networks. Neuroimage 21:954-964

44. Moratti S, Rubio G, Campo P, Keil A, Ortiz T (2008) Hypofunction of right temporoparietal cortex during emotional arousal in depression. Arch Gen Psychiatry 65:532-541

45. Ray WJ, Odenwald M, Neuner F, Schauer M, Ruf M, Wienbruch C, Rockstroh B, Elbert T (2006) Decoupling neural networks from reality: dissociative experiences in torture victims are reflected in abnormal brain waves in left frontal cortex. Psychol Sci 17:825-829

46. Regan D (1989) Human Brain Electrophysiology: Evoked Potentials and Evoked Magnetic Fields in Science and Medicine. Elsevier, New York

47. Rief W, Hiller W (2003) A new approach to the assessment of the treatment effects of somatoform disorders. Psychosomatics 44:492-498

48. Rossignol M, Philippot P, Douilliez C, Crommelinck M, Campanella $S$ (2005) The perception of fearful and happy facial expression is modulated by anxiety: an event-related potential study. Neurosci Lett 377:115-120

49. Sheehan DV, Lecrubier Y, Sheehan KH, Amorim P, Janavs J Weiller E, Hergueta T, Baker R, Dunbar GC (1998) The MiniInternational Neuropsychiatric Interview (M.I.N.I.): the development and validation of a structured diagnostic psychiatric interview for DSM-IV and ICD-10. J Clin Psychiatry 59(Suppl 20):22-33 quiz 34-57

50. Sloan DM, Strauss ME, Quirk SW, Sajatovic M (1997) Subjective and expressive emotional responses in depression. J Affect Disord 46:135-141

51. Sloan DM, Strauss ME, Wisner KL (2001) Diminished response to pleasant stimuli by depressed women. J Abnorm Psychol 110:488-493

52. vivo (2008) http://www.vivo.org

53. Williams JB (1988) A structured interview guide for the Hamilton Depression Rating Scale. Arch Gen Psychiatry 45:742-747 